\title{
Towards Improving Educational Decisions by Utilizing Interdisciplinary Research
}

\author{
Joseph Klein $^{1}$ \\ ${ }^{1}$ School of Education, Bar-Ilan University, Ramat-Gan 52900, Israel \\ Correspondence: Joseph Klein, Bar-Ilan University, Ramat-Gan 52900, Israel.
}

Received: July 9, 2014 Accepted: July 23, 2014 Online Published: August 19, 2014

doi:10.11114/jets.v2i4.494 URL: http://dx.doi.org/10.11114/jets.v2i4.494

\begin{abstract}
Recent neurophysiological advances may support the advisability of delaying decisions when possible and practical. An empirical study, based on an educational dilemma, compared the outcome of postponing an educational decision overnight or for a longer period.

340 teachers read a report on an educational dilemma and gave an immediate opinion. About half of the group reviewed their decision after an overnight delay; the rest did so after a month. Participants were not involved with the dilemma between sessions. The cognitive aspects of the question addressed by participants in the immediate and delayed decisions were compared.
\end{abstract}

After a month's delay, more cognitive aspects were utilized than in the decision taken after one day. The immediate decision was least comprehensive.

Postponement of educational decisions offers the opportunity to utilize a richer variety of cognitive sources.

Keywords: Choice, conscious, decision making, unconscious

\section{Theoretical Background}

Introduction

In schools and in the upper echelons of the education system, decisions are made daily. In retrospect, some of these decisions were wisely made and some less so. The maximization of judicious decision making has generated considerable research. Problem solving, learning and decision making processes were studied by psychologists and other social science researchers. They constructed theoretical models based on the observations of decision makers using many experimental designs. Hypothetical neurological data-processing pathways were posited by researchers but were never proven. However, in the last few decades interdisciplinary studies have yielded new insights into biochemical and bio-psychological processes in the brain. In addition, these studies shed light on the learning potential and the limits of learning, memory and decision making. They have shown that when confronted with new information, the brain processes it in a stepwise fashion and not immediately. These studies also show that better implantation improves the decisions made using the information. If implantation is done gradually, then it would be wiser to delay certain decisions until the data are fully processed by the brain. This is normally the case when new educational dilemmas arise.

Postponing the decision is not always easy. The quandary of whether to respond immediately, or to delay a response, must take into consideration the intensive and dynamic organizational structures that exist in educational institutions. Dilemmas are constantly arising for which both internal and external elements anticipate swift answers. However, regardless of the pressures exerted on the decision makers, it is preferable in many cases to delay the decision and increase the chances of making the right choices.

\section{Literature Review}

Cognitive and non-cognitive processes in learning and decision making

Rapid learning and decision making processes

The cognitive theory describes learning and memory as developmental occurrences which begin by receiving new information via sensory inputs, which are transmitted to the brain. There it undergoes an initial coding and sensory 
impression analysis. Later, a consolidation of this information is formed, strengthened and stored in the long term memory (LTM). This allows the stored datum to be recalled and used in the future. While the LTM is being formed, the information is further processed beyond the initial stages (Faw, 2003; Singer, 2006; XiaoHua, 2010). Storing memories in the LTM consists of unconscious incremental neurobiological changes in the brain (Stickgold, 2005; Diekelman et al., 2009; Karni et al., 2009). Since it takes time for full integration, it was hypothesized that delaying a response to new information would be advantageous.

This hypothesis was tested by giving participants a problem to solve immediately. Thereafter, they were distracted from the assignment by receiving an additional task. When they finished the task, they were asked to revisit the original dilemma and solve it again. The initial and delayed answers were later compared. Calvillo and Penaloza (2009) reported no significant change in the quality of the two responses and concluded that unconscious thought processes do not improve decision making. However, a relatively large number of studies showed an improvement in the subsequent decision (Dorfman et al. 1996; Segal, 2004; Dijksterhuis, 2004; Dijksterhuis et al., 2006). This was attributed to the effect of neurological actions on memory, and to unconscious thought processes such as incubation (Dijksterhuis et al., 2006).

Incubation is defined as an unconscious replacement process of thoughts that were consciously evoked at a certain point in time and became a new insight at some later point in time (Seabrook and Dienes, 2003). Incubation, as its name implies, is a period of time in which unconscious thought processes are allowed to develop slowly for problems that are bothering the individual. The conscious mind becomes aware of these processes only when a solution is formed. In a meta-analysis that analyzed the findings of many studies in which the solution to a given problem was delayed by a period of time of up to 20 minutes, it was found that incubation enhances problem solving. Divergent thinking tasks benefited more from the incubation period than linguistic and visual insight tasks.

Surprisingly, when solving linguistic insight problems the incubation effect was stronger when the subjects were engaged in low cognitive demand tasks, rather than rest, during the incubation period (Sio \& Ormerod, 2009). Research into delayed decision making has been conducted primarily in the fields of psychology and economics, and used problems that have only one correct answer. The ability to improve upon decision making by delaying the decision has not been studied in education in which the majority of the problems do not have a single unique correct answer and many legitimately correct decisions are possible for a given situation.

\section{Ongoing Processes of Learning and Decision Making}

A number of researchers investigated whether assimilating data into the memory takes longer than a few minutes. These researchers explored the benefits of an overnight delay in making a decision on new information. Until the 1950's it was thought that one of the main purposes of sleep is to prevent new input that might cause the deletion of previously incorporated data. Neurologists later discovered that the brain is active during sleep, transferring new information from the short term memory (STM) to the LTM (Karni et al., 1994). It was also found that different stages of sleep assimilate different informational components in the brain (Stickgold, 2006; Rauchs et al., 2005). During the first sleep stage, intellectual and emotional information is distilled. Subsequently, it is cataloged to a specific section in the memory for future access. The deep sleep stage is used for the long term implementation of learned facts such as names of places. The rapid eye movement (REM) phase is used to improve differentiation between objects (Karni et al., 1994). Sleep allows for the successful execution of complex processes that would be difficult to execute correctly when a person is awake.

In one experiment, subjects were asked to memorize a sequence of random nonsense notes on a keyboard. The subjects were asked to repeat the sequence, once immediately after learning it and the second time after a night's sleep. The subjects remembered the sequence better after a night's sleep (Nishida and Walker, 2007). In another experiment, the subjects learned how to solve a mathematical problem in a long and tedious manner. There was then a break in which the subjects were asked not to sleep and to practice the arithmetic technique intensively. The researchers intentionally did not tell the subjects that there is an easier way to solve the problem. After the break the subjects were asked to solve problems similar to those that they drilled. Some of the subjects solved the problems using the easier method. A parallel group of subjects were given identical problems and were taught the same difficult method. These subjects were requested to sleep during the break. The results showed that more subjects from the sleep group found the easier way to solve the problems. The brains of these subjects seemed to have discovered a simpler way to solve the problems without being told that such a method exists (Wagner et al., 2004).

There are those who hypothesize that during sleep hidden connections are found between memories that are not apparent during the waking hours (Savage and West, 2007). However, there is no proof that the brain during sleep can access memories by a unique pathway not available to the brain during waking hours. One theory as to why these processes occur during sleep is that certain cognitive resources, needed to process memories in the LTM, are in use for 
other purposes by the brain during the waking hours. This impedes the coding and processing of data to the LTM (Stickgold, 2006). Even a nap during the day can help in processing the data to the LTM on condition that dream-phase sleep has been attained (Censor et al., 2006). However, normal night sleep has been shown to be more effective.

Sleep improves cognitive processes but it does not improve motor skills. In a study in which improvements in motor skills were compared, no significant differences were found between the subjects who slept between tasks and those who did not (Nemeth et al., 2010). All the studies mentioned above were done using problems that have defined correct answers. Nothing has been reported with regard to problems that have many legitimate correct answers or involve probabilistic decision situations.

\section{Long Term Learning Processes and Decision Making.}

Delayed decisions are also influenced by conscious reflective processes. The individual is able to review the initial decision, its motives, benefits and limitations critically and thereby arrive at a better conclusion. A body of research has shown the significance of reflective and meta-cognitive processes in improving thinking (Brown, 1987; Kaniel, 2001; Koriat, 2007, 2008). Although psychological and neurological studies dealing with improving LTM and learning have used time intervals of a few minutes to an overnight sleep (Sio and Ormerod, op cit), reflective and meta-cognitive studies have used longer delays (Kaniel, 2001). In these studies, decisions are often changed over long periods of time even when no new external data influence these processes. Experiments were conducted measuring improvement in judgment after a period longer than overnight (Mazursky \& Ganzach, 1998; Mazursky, 2000). In one case, consumers evaluated a product and one week later was asked their opinion about an identical item. The researchers found that the delayed assessment was better. This experiment lacked a shorter time-interval control such as a few hours or the day after the initial decision. Therefore, it is difficult to judge the optimal time required to arrive at a better conclusion. With that said, the existence of long term reflective processes gives rise to a theory, which is tested herein, that delayed decisions improve with time even if there are no new external data. This phenomenon is also seen when authors edit and reedit their own work.

\section{Probabilistic Decisions}

The research done on delayed decision making used problems that by consensus have answers whose veracity can be objectively quantified. Such research has not been conducted in education where the outcomes of the decisions are not apparent immediately. In such cases, the degree of improvement, if any, as a result of the delay, can be known only at some future point in time. However, with the use of suitable quantifiable measuring tools, the psycho-biological processes theorized for the improvements seen in delayed decisions should be applicable in this type of deliberation as well. In such cases, effectiveness can be determined by measuring the quality of the decision making process (Morera and Budescu, 2001).

In the literature there are models that detail the main cognitive aspects of reaching probabilistic decisions that have systemic orientations. These models have certain elements in common including: (a) systemic information gathering to clarify dilemma that need to be resolved, and to determine the preferred goal; (b) setting independent alternatives for achieving the aim or for solving the problem at hand, and developing criteria or attributes for comparing the alternatives; (c) choosing the alternative or alternatives that received the highest score (Simon, 1991, 1993; Kaniel, 2009). The quality of the decision making is based on the number of elements used by the decision maker to arrive at the decision. The process is considered to be more deliberately systematic if there are a greater number of elements used.

Using consciously determined criteria to explore different processes of which some might be unconscious is in line with the current literature which states that unconscious thought processes, after a certain incubation period, result in new conscious processes and understandings. In light of what is known in the literature, our research will test a hypothesis that delaying a decision in an educational dilemma for periods of days and weeks will ultimately allow the decision maker to take into account a greater number of elements in the decision making than if the decision had to be made immediately. Mazursky and Ganzach (1998) and Mazursky (2000) reported that a delay of one or two weeks improved consumer decision making. However, they did not check the quality of the decision making after a night's sleep. Therefore, we must compare between the qualities of three probabilistic decisions, one made immediately, another after a night's sleep, and a decision made at a later point in time. Confirmation of this hypothesis will allow the laying of a foundation for additional studies which will measure the partial contributions of conscious and unconscious thought processes in decision making.

\section{Methodology}

\section{Subjects and procedures}

A sample size of 340 high school teachers from 68 different schools was taken. The schools were from different socio-economic strata, from both urban and rural schools and schools located in different geographical areas within 
Israel.

Each teacher was presented with an educational dilemma and asked to make an immediate decision and a second one about the same matter at a later time point. The immediate response was written on a page with no limits on the length of the answer or on the time that it took to write it. This was done to allow maximum individualistic expression of their thought process. One day later, 165 of the teachers were asked to review the dilemma and to reweigh their decision. Since none of the teachers knew at the time of the first meeting that they would be asked to repeat the process, they could not have planned their second decision in advance. The other group of 175 teachers was asked to reconsider the original conclusion after 30 days. As with the first group, they were unaware that they would be asked to revisit their decision.

The second decision for both groups involved a review of both the dilemma and their original written judgment. They were told that they could leave the first decision as is or change it as they see fit. We did not give them the option of writing a totally new decision without seeing the original response. One reason for not allowing this option was the extra work involved in completely rewriting their conclusion. In a pre-test that we conducted, participants were willing to write their first immediate decision. However, when they were asked to repeat this task after a delay, many of the participants declined to write a second decision due to the amount of work involved. On the other hand, they did not object to expressing their second opinion by reading their previous work and correcting it by adding or crossing out certain parts or by writing an addendum at the end of the original opinion. The other reason for conducting the experiment in this manner was that it more authentically resembles the real world process in which a person recalls the previous decision before making a different one.

After the participants finished the revision of the original answer, they were asked if they thought about the dilemma in the period between the two decision making points. They were also asked if they were exposed to new information that was relevant to the dilemma or if they discussed it with their colleagues. A small number of the participants replied affirmatively to one or more of these questions. In the statistical analysis we included only those 340 subjects who did not consciously think about the dilemma or discuss it between the two decisions. Their answers were analyzed to ascertain the number of theoretical decision making components used. The number of components was compared between the first and second decision. The statistical measurements used will be discussed further.

\section{The Educational Dilemma}

The case and the criteria for assessing responses (Appendix A) are based on Klein (1999) with minor alterations. The dilemma described a dominant Parent's Committee that demanded that the school change the teaching methods in a number of subjects. The principal decided to discuss this demand with the educational staff at the upcoming staff meeting and come up with a proper response.

Data analysis provided a more in-depth examination of whether the participants' responses at each interval (in both immediate and delayed decisions) referred to three components of the decision making process and their subcomponents: (a) gathering data pertaining to the school's main aims, needs, and challenges; (b) determining alternatives for resolving the case and choosing attributes for comparing them;, and (c) selecting a decision by choosing the alternative that had the highest rating by comparison.

As seen in Appendix A, the component of data-gathering contains nine subcomponents within three domains: (a) examination of all of the school's programs and its priority-setting policy (one subcomponent); (b) collecting data about the scholastic level of English and Mathematics in the school (four subcomponents;) and (c) gathering information about the effectiveness of computers in improving learning (four subcomponents).

The component of alternative selections included four subcomponents: (a) objective tools and the professional literature; (b) experiences of colleagues in other schools; (c) consultations with school-related personnel such as teachers, supervisors, and parents; and (d) organizing the alternative options into a list of priorities.

In the third component, involving the final decision, there were two subcomponents: (a) comparison of alternatives; and (b) selection of the most suitable alternative as the final choice.

If a participant did not relate at all to a certain component, it was marked as " 1 " which represents a lack of consideration of that specific component. If the participant related to a component it was marked as " 2 ". Even if the participant referred only to some, but not all of the aspects of a component, it was marked as " 2 ". We purposely did not give an internal hierarchical order to the subcomponents due to a lack of consensus on such an order. In the component "sharing in decision making" there was a consensus that the participant's awareness of this component was a direct function of the number of relevant educational sources that the participant utilized in making a decision. Therefore, this was defined as a continuous variable.

\section{Selection of Criteria for Analysis}


These criteria are not the only ones by which this case can be analyzed. Other educational and value-oriented aspects could also be used (Begley \& Johansson, 1998). However, the criteria selected for this work are sufficient in determining whether any changes occurred between the participants' stance about the case immediately after exposure and after a given delay.

\section{Calibration}

A sample of six responses submitted by the participants was examined independently by three evaluators, who held executive positions in their schools, had a Master's degree in education, and were not included in the groups of participants. They were instructed to grade the answers with reference to the selected criteria. A response was defined as relating to each of the criteria when it contained a clearly articulated statement referring to the criterion. After completing the work, the three compared their rankings in order to reach a consensus about the evaluation and adjusted their expectations. They then examined an additional four responses independently, and found that the evaluations were very similar. Following a triangulated agreement, they continued to examine the remaining responses of the participants.

\section{Findings}

In order to ascertain the effect of a time delay on the cognitive process of educational decision making, we performed a 2X2 two way MANOVA, in which there were two time points (immediate and delayed) and two groups (one with a delay of 24 hours and the other after 30 days). In analyzing the procedures used by participants at different time points, three main components of the cognitive decision-making process were examined. These were: (a) gathering information for better understanding of the problem; (b) putting forward alternative solutions; (c) decision making. We also examined the extent to which the participants involved the educational staff in reaching their conclusions

There were statistically significant differences in the MANOVA analyses according to the factor of time in general for all the components measured, and also in the interaction between the time factor per se and the group, i.e. length of the delay. The analyses were initially controlled for the educational level of the participants, their positions in their schools, scope of experience in education, and gender. None of these control factors showed any differences in the analysis and therefore they were removed from the reported results.

There was a significant difference in the time element in measuring before and after in both groups $\mathrm{F}(3,336)=31.41$, $\mathrm{p}<.00, \eta^{2}=.22$. The interaction between the factor of time and the group element was also statistically significant $(3,336)$ $=12.63, \mathrm{p}<.00, \eta^{2}=.10$. This shows that there was an effect in the time delay as well. There were also significant differences between the two groups in the decision making components $F(3,336)=21.15, p<.00, \eta^{2}=.16$.

Table 1 presents an analysis of the results of the two decisions.

Table 1. Averages and standard deviations of the results of the immediate and delayed decisions

\begin{tabular}{llll}
\hline & Mean & S.D. \\
\hline $\begin{array}{l}\text { Second decision after a 24 } \\
\text { hour delay }\end{array}$ & $\begin{array}{l}\text { Results of the initial } \\
\text { response } \\
\text { Results of the response } \\
\text { after 24 hours }\end{array}$ & 1.25 & 0.18 \\
$\begin{array}{l}\text { Second decision after a 1 } \\
\text { month delay }\end{array}$ & $\begin{array}{l}\text { Results of the initial } \\
\text { response } \\
\text { Results of the response } \\
\text { after 1 month }\end{array}$ & 1.35 & 0.19 \\
\hline
\end{tabular}

The range of the answers fell between 1, representing lack of relation to that specific cognitive component in the decision making process and 2 , indicating that the participant related to that component. The univariate analysis results on each of the cognitive decision making components are presented in Table 2 which includes both averages and standard deviations along with the results $(\mathrm{F})$. 
Table 2. Averages, standard deviations, and $\mathrm{F}$ analyses of three components of the decision-making process, for decisions made immediately and after one day and one month

\begin{tabular}{|c|c|c|c|c|c|c|c|c|}
\hline & & \multicolumn{2}{|c|}{ Immediate } & \multicolumn{2}{|c|}{ After } & \multirow{4}{*}{$\mathrm{F}$ (time) } & \multirow{4}{*}{$\mathrm{F}$ (Interaction) } & \multirow{4}{*}{ F (Group) } \\
\hline \multirow[t]{3}{*}{ Variable } & & Group & Group & Group & Group & & & \\
\hline & & & & NO. 1 & NO. 2 & & & \\
\hline & & & & $\begin{array}{l}\text { After } \\
24 \text { hours }\end{array}$ & $\begin{array}{l}\text { After } 1 \\
\text { month }\end{array}$ & & & \\
\hline \multirow[t]{3}{*}{ Data collection } & $\mathrm{M}$ & 1.22 & 1.23 & 1.24 & 1.44 & $80.02 * * *$ & $32.89 * * *$ & $54.54 * * *$ \\
\hline & $S$ & 0.17 & 0.20 & 0.18 & 0.22 & $\mathrm{H}^{2}=0.19$ & $\eta^{2}=0.08$ & $\mathrm{H}^{2}=0.14$ \\
\hline & $\mathrm{N}$ & 165 & 175 & 165 & 175 & & & \\
\hline \multirow[t]{3}{*}{ Alternative } & $\mathrm{M}$ & 1.26 & 1.41 & 1.31 & 1.51 & $60.02 * * *$ & $7.17^{* * *}$ & $44.45 * * *$ \\
\hline & $\mathrm{S}$ & 0.24 & 0.25 & 0.27 & 0.25 & $\mathrm{H}^{2}=0.15$ & $\eta^{2}==0.02$ & $\mathrm{H}^{2}=0.12$ \\
\hline & $\mathrm{N}$ & 165 & 175 & 165 & 175 & & & \\
\hline \multirow[t]{3}{*}{ Decision } & $\mathrm{M}$ & 1.33 & 1.37 & 1.36 & 1.50 & $42.65 * * *$ & $18.36 * * *$ & $6.21 *$ \\
\hline & $\mathrm{S}$ & 0.33 & 0.36 & 0.33 & 0.34 & $\eta^{2}=0.11$ & $\eta^{2}=0.05$ & $\eta^{2}=0.02$ \\
\hline & $\mathrm{N}$ & 165 & 175 & 165 & 175 & & & \\
\hline
\end{tabular}

Group 1 reconsidered their decision after 24 hours and group 2 after 1 month.

We also examined in a similar fashion the extent to which the participants involved the educational staff in the decision making plans, both immediately and after a delay. A 2X2 two way MANOVA analysis of variance was performed, with two time points (immediate and after a time delay) and two groups (one group with a time delay of 24 hours and the other with a delay of a month). We found that the time element was statistically significant $F(3,336)=40.22, p<.00$, $\eta^{2}=.11$. In addition, the interaction between the time measurement component and the group was found to be statistically significant $\mathrm{F}(3,336)=9.71, \mathrm{p}<.00, \eta^{2}=.003$. Table 3 presents the averages and the standard deviation for this analysis.

Table 3. Averages and standard deviations with respect to degree of involvement of staff in immediate and delayed decisions

\begin{tabular}{lllll}
\hline Group & & Mean & S.D. & N \\
\hline $\begin{array}{l}\text { The extent of involving the educational staff before the } \\
\text { delay }\end{array}$ & Group No. 1: 24 hours & .91 & .86 & 165 \\
& Group No. 2, 30 days & 1.08 & .96 & 175 \\
& & & & \\
The extent of involving the educational staff after the delay & Group No. 1: 24 hours & 1.03 & 1.01 & 165 \\
& Group No. 2, 30 days & 1.47 & 1.07 & 175 \\
\hline
\end{tabular}

The answers ranged from 0-4, where 0 signifies that the participant reached the decision without any input from external sources ( 0 external sources) and 4 represents the participation of 4 external sources: school system supervisors, parents, teachers and students.

A post Hoc analysis shows that there were no significant differences in the measurement, with regard to the immediate decision, between the group that made a delayed decision after 24 hours $(\mathrm{M}=0.91)$ and the second group whose delayed decision took place after 1 month $(\mathrm{M}=1.08)$. A repeated measurement, after the delay, found statistically significant differences between the two groups. The 24 hour group showed a lesser tendency to involve external sources in their decision making $(\mathrm{M}=1.03)$ as compared with the 1 month delay group $(\mathrm{M}=146)$.

\section{Discussion}

Considerable efforts have been made in improving decision making methods in education and in other fields as well. The assumption made is that implementing these techniques will empower human judgment and optimize decision making. The interdisciplinary research in this field has contributed to the realization of this challenge by cautiously connecting the psychological aspects of the thought process to the biological ones. 
The first observations that delay improves judgments were the result of human experience rather than research. This universal understanding can be seen in idioms from many different languages. In English, the term "sleep on it overnight", in French "Dormir là-dessus durant la nuit", in Italian "Dormire su di essa durante la note", in Spanish "Duerma toda la noche", in German "Schlafen auf ihn über Nacht" in Arabic "النوم على ذلك بين عشية وضحاها" in " Russian "Спать на нем всю ночь" all portray the same thought. The term "on second thought" is also prevalent in many other languages and it indicates improvement of the initial judgment.

The change seen in the delayed effect was later explained by cognitive psychologists and neurobiologists as a process in which new information is embedded in the LTM gradually and not immediately upon exposure to the new information. Only after the storing of the information in the LTM can it be effectively utilized for the purposes of judgment and decision making (Faw and Singer 2003, 2006). The research done on this storage process and its influence on the quality of judgment has focused on brief delays, from between a few minutes to overnight. In addition, the emphasis of most of this research has been on single variable decisions, whose prevalence is insignificant in the educational realm. Little is known about the value of delaying a decision for more than a day (Mazursky and Ganzach 1998; Mazursky, 2010). However, the educational field is characterized by tasks that can span weeks, months and sometimes, years. This facilitates a stepwise decision process rather than a onetime decision in cases where a delayed conclusion is shown to be more effective.

Our study is unique in that it uses probabilistic educational decisions that have many legitimate answers. It also shows that delaying a decision for more than a day increases the application of cognitive components that are theoretically essential for improved decision making. The term "improved decision making process" is used by us to indicate that the decision maker relates more successfully to the most relevant components, rather than to all of the associated criteria. In a broader context, this issue should be examined in respect to other educational concepts. All these areas require the processing of information, learning and knowledge to assess various action options.

After a day's delay there is an increase in the teachers' attitude to the components of "gathering educational information", "assessing alternative options" and "involving relevant school and interested parties". There was no improvement in the "decision-making" component, which is the last step in the probabilistic process. After a month's delay there is an additional rise in the number of decision making components used as well as an improvement in the last decision making component that was not evident during the previous round. We do not know when the exact time of increased awareness occurs in the different components between one day and a month. It would be interesting to investigate in a future study whether these changes happen gradually throughout the month or if there is a specific window of time in which these processes take place.

It is important to point out that the increase in the number of decision making components used by the participants is on the one hand statistically significant but on the other hand of confined scope and intensity. Therefore, the approach to improvement of decision making outlined in this study should not be seen as a replacement to other proven methods but as an additional tool that is different in its characteristics from many other cognitive techniques described in the literature.

Our findings show that there is an improvement in educational decision making after a one-night delay but the mechanisms have not been elucidated. There is no biological evidence in the brain research literature that the LTM continuously strengthens for periods of days and weeks. From the psychological perspective, this change can be explained in various ways (Sio and Ormerod, 2009). We can attribute the improvement to the decision maker's unconscious exposure to external knowledge that served to reveal more cognitive components. The participants reported that they were not exposed to any additional relevant knowledge between the first decision and the second one and that they did not consciously think about the decision that they made between the two decision making intervals. However, we had no way of insuring the veracity of these reports, especially in the 1 month delay group. It is quite possible that creative and cognitive processes are involved. Bos, Dijksterhuis and Baaren (2008) indicate that the conscious mind's "decision" to acquire more information in the future is an essential condition for unconscious brain activity. Therefore, if we conclude that the improvement in the repeated decision was due to unconscious brain activities we must make the assumption that the participants viewed the information given at the time of the initial decision to be relevant for the future as well.

Whatever the reasons may be, the results show that there is an improvement and enrichment in the decision making process over time as compared to the initial decision even though no conscious cognitive effort is made during the decision making interval. Improvements are not only achieved by initiated cognitive interventions but also in the abstention of such activities. These two processes do not contradict each other but rather complement each other. Both the cognitive and unconscious interventions contribute towards improving the decision making process, and more research is needed to find the optimal balance between the two. 
Our findings can be implemented in improved teaching strategies. The lesson's "take home" messages should be implemented in a stepwise fashion with a break between each learning unit. In addition, the current lesson should go over the previous lesson's main points, assuming that there was a break between the lessons. Intensively "cramming" information in concentrated daily learning sessions impedes the assimilation of the imparted knowledge. This is due in part to the limited storage capacity of the STM, where new data must be held before input into the LTM (Korman et al. 2007).

This delay in decision making has implications in a teacher's role as a disciplinarian as well. The teacher may react immediately to abhorrent behavior and later, on second thought, realize that there was a better way.

Schools are often under pressure by supervisors, parents, or interested parties to respond immediately to their demands, and it is not always possible to delay action. The result may be a hasty, inadequate solution. Sometimes the pressure is internal. Staff members prefer to deal with matters in one sitting and avoid additional sessions. Fostering a culture that favors quality decisions will go a long way in minimizing instinctive, hastily made decisions. If the internal and external pressure groups that the school has to deal with realize that the school's educational staff are delaying their decisions to maximize the cognitive and unconscious benefits that delayed decision making provides, they will, hopefully, tend to reduce the pressure.

There is tension between rapid human progress which is often superficial and a slower but more thorough approach to human advancement. Normative and empirical research is needed to find the circumstances that are advantageous to accelerate or slow down the rate of advancement. Such insights will allow for a better understanding of how to deal with the continuum between the two poles.

\section{Conclusion}

If it was thought that research into decision making reached a saturation point, interdisciplinary understandings have paved new research vistas. The synergy between biology and psychology has given deeper understandings in areas where they intersect. Every so often there are concerns voiced that psychology and education will become subservient to biochemical mechanisms which will reduce the individual's capabilities to probabilistic and deterministic variables. Many students encouraged by great educators have reached goals and achievements far beyond what experts in other fields predicted. Therefore, the independence of the educational field must be preserved. However, this does not exempt educators from availing themselves of the benefits of interdisciplinary research and knowledge.

\section{References}

Begley, P. T., \& Johansson, O. (1998). The Values of School Administration: Preferences, Ethics, and Conflicts. Journal of School Leadership, 9(4), 399-422.

Bos, M.W., Dijksterhuis, A., \& Van Baaren, R.B. (2008). On the goal-dependency of unconscious thought. Journal of Experimental Social Psychology, 44(4), 1114-1120. http://dx.doi.org/10.1016/j.jesp.2008.01.001

Brown, A. L. (1987). Metacognition, executive control, self-regulation, and other more mysterious mechanisms. In F. E. Weinert. \& R. H. Kluwe (Eds.), Metacognition, motivation, and understanding (pp. 65-116). Hillsdale, NJ: Lawrence Erlbaum

Calvillo, D. P., \& Penaloza, A. (2009). Are complex decisions better left to the unconscious? Further failed replications of the deliberation-without-attention effect. Judgment and Decision Making, 4(6), 509-517.

Censor, N., Karni, A., \& Sagi, D. (2006) A link between perceptual learning, adaptation and sleep. Vision Research, 46(23), 4071-4074. http://dx.doi.org/10.1016/j.visres.2006.07.022

Diekelman, S., Wilhelm, I., \& Born, F. (2009). The whats and whens of sleep-dependent memory consolidation, Sleep Medicine Reviews, 13(5), 309-321. http://dx.doi.org/10.1016/j.smrv.2008.08.002

Dijksterhuis, Ap. (2004). Think Different: The Merits of Unconscious Thought in Preference Development and Decision Making. Journal of Personality and Social Psychology, 87(5), 586-598. http://dx.doi.org/10.1037/0022-3514.87.5.586

Dijksterhuis, A., Bos, M.W., Nordgren, L.F., \& van Baaren, R.B. (2006). On making the right choice: The deliberation-without-attention effect. Science, 311(5763), 1005-1007. http://dx.doi.org/10.1126/science.1121629

Dorfman, J., Shames, V. A.M., \& Kihlstrom, F. (1996). Intuition, incubation and insight: Implicit cognition in problem solving. In Underwood, G. (Ed.).Implicit cognition, Oxford Science Publications. pp: 257-296.

Faw, B. (2003). Pre frontal executive committee for perception, working memory, attention, long term memory, motor control and thinking: A tutorial review, Consciousness and Cognition. 12(1), 83-139. http://dx.doi.org/10.1016/S1053-8100(02)00030-2 
Kaniel, S. (2001). The psychology of control of cognition. Ramat Gan: Bar-Ilan University.

Kaniel, S. (2009). The courage to decide and act: decision making by individuals and groups. Tel Aviv University: Ramot.

Karni, A., Tanne, D., Rubenstein, B. S., Askenasy, J. J. M., \& Sagi, D. (1994). Dependence on REM sleep of overnight improvement of a perceptual skill. Science 265(5172), 679-682. http://dx.doi.org/10.1126/science.8036518

Karni A, Ungerleider LG, Carrier J (2009) Contribution of night and day sleep versus simple passage of time to the consolidation of motor sequence and visuomotor adaptation learning. Experimental Brain Research, 195(1), 15-26. http://dx.doi.org/10.1007/s00221-009-1748-y

Klein, J. (1999). The relationship between level of academic education and reversible and irreversible process of probability decision making. Higher Education, 37(4), 323-339. http://dx.doi.org/10.1023/A:1003608412240

Koriat, A. (2007). Metacognition and consciousness. In P. D. Zelazo, M. Moscovitch, \& E. Thompson (Eds.), The Cambridge Handbook of Consciousness (pp. 289-325). New York: Cambridge University Press . http://dx.doi.org/10.1017/CBO9780511816789.012

Koriat, A. (2008). Easy comes, easy goes? The link between learning and remembering and its exploitation in metacognition. Memory \& Cognition, 36(2), 416-428.

Korman, M., Doyon, J., Doljansky, J., Carrier, J., Dagan, Y., \& Karni. A. (2007). Daytime sleep condenses the time course of motor memory consolidation, Nature Neuroscience 10(9), 1206 - 1213. http://dx.doi.org/10.1038/nn1959

Mazursky, D. (2000). The Effects of Time Delays on Consumers' Use of Different Criteria for Product Purchase Decisions. Journal of Business and Psychology, 15(1), 163-175. http://dx.doi.org/10.1023/A:1007779121123

Mazursky, D., \& Ganzach, Y. (1998). Does involvement moderate time-dependent biases in consumer multi attribute judgment? Journal of Business research, 4l(2), 95-103. http://dx.doi.org/10.1016/S0148-2963(97)00016-7

Morera, O. F., \& Budescu, D. V. (2001). Random error in analytic hierarchies: a comparison of holistic and decompositional decision strategies. Journal of Behavioral Decision Making, 14(3), 223-242. http://dx.doi.org/10.1002/bdm.374

Nemeth, D., Janacsek, K., Londe, Z.S., Ullman, M. T., Howard D. V., \& Howard, J. H. Jr. (2010). Sleep has no critical role in implicit motor sequence learning in young and old adults. Experimental Research Brain, 201(2), 351-358. http://dx.doi.org/10.1007/s00221-009-2024-x

Nishida, M., \& Walker, M.P. (2007). Daytime Naps, Motor Memory Consolidation and Regionally Specific Sleep Spindles. PLoS ONE, 2(4): e341. http://dx.doi.org/10.1371/journal.pone.0000341

Rauchs, G., Desgranges, B., Foret, J., \& Eustache, F. (2005). The relationships between memory systems and sleep stages. Journal of Sleep Research, 14(2), 123-140. http://dx.doi.org/10.1111/j.1365-2869.2005.00450.x

Savage, V. M., \& West, G. B. (2007). A quantitative, theoretical framework for understanding mammalian sleep. Proceedings of the National Academy of Sciences, 104(3), 1051-1056. http://dx.doi.org/10.1073/pnas.0610080104

Seabrook, \& Dienes. (2003). Incubation in problem solving as a context effect. Proceedings of the 25th Annual Meeting of the Cognitive Science Society, Boston, Massachusetts, USA.

Segal, E. (2004). Incubation in insight problem solving. Creativity Research Journal, 16(1), 141-148. http://dx.doi.org/10.1207/s15326934crj1601_13

Simon, H.A. (1993). Decision making: Rational, nonrational, and irrational. Educational Administration Quarterly, 29(3), 392-411. http://dx.doi.org/10.1177/0013161X93029003009

Simon, H. (1991). Bounded rationality and organizational learning. Organization Science, 2(1), 125-134. http://dx.doi.org/10.1287/orsc.2.1.125

Singer, T. (2006). The neuronal basis and ontogeny of empathy and mind reading: Literature and implications for future research. Neuroscience \& Biobehavioral, 30(6), 855-863. http://dx.doi.org/10.1016/j.neubiorev.2006.06.011

Sio, U., \& Ormerod, T.C. (2009). Does incubation enhance problem solving? A meta-analytic review. Psychological Bulletin, 135(1), 94-120. http://dx.doi.org/10.1037/a0014212

Stickgold, R. (2005). Sleep-dependent memory consolidation. Nature, 437(7063), 1272-1278. http://dx.doi.org/10.1038/nature04286

Stickgold, R. (2006). Neuroscience: A memory boost while you sleep. Nature, 444(7119), 559-560. http://dx.doi.org/10.1038/nature05309 
Surowiecki, J. (2004). The Wisdom of Crowds: Why the Many Are Smarter Than the Few and How Collective Wisdom Shapes Business, Economies, Societies and Nations Little, Brown http://dx.doi.org/10.1038/nature02223

Wagner, U., Gais, S., Haider, H., Verleger, R., \& Born, J. (2004). Sleep inspires insight. Nature, 427(6972), 352-355.

XiaoHua, Z. (2010). The study of the framework of intelligence information processing. Applied Mechanics and Materials, 20(3), 861-866.

\section{Appendix A}

\section{The event}

Please read the following description of a hypothetical event, and answer the questions with regard to that episode on the blank sheets attached to these pages.

In the "Hadarim" school there is a long standing tradition of strong intensive parental involvement in the school. In certain years the Parent's Committee contributed greatly to the school's development. In other years the committee was characterized by a domineering attitude in an effort to dictate institutional policies and methods of implementation. This caused more than once the resignation of the school's principals who felt that their freedom of action was severely curtailed. Very shortly after Dan Arzi became principal of Hadarim, he received this letter from the Parent's Committee:

Dear Sir:

We wish to request that in the next school year approximately one-quarter of the total teaching hours may be allocated to intensified, computer-assisted studies in English and in mathematics. We trust that this step will solve the problem of the low level of instruction in these subjects in our school. In other institutions in the region, computers are already employed for such purposes. There is no good reason for us to adhere to the old systems. We remind you that parents have a legal right to determine the content of one-quarter of the school program.

Use of computers in the school will undoubtedly improve the level of achievement in English and mathematics. An allocation from the regional department of education for acquisition of computers is essential. The parents will also assist in raising funds.

Adaptation of the teaching plan according to our request will require a change in personnel and certain other adjustments. It will be necessary to curtail the number of study hours in several fields, and to employ new staff members with the appropriate skills in teaching English and mathematics.

We will be happy to help as much as possible in the success of the suggested project. Considering the importance of the undertaking and the fact that parents do have a legal right to exert influence on program planning, we very much hope that the proposal will be implemented.

Sincerely,

A. Levi

Chairman of the committee

Dan felt that he was still too new to this school to respond, and therefore he decided to present this letter at the next educational staff meeting and discuss with the school's educational staff to formulate an appropriate answer to the letter received from the Parent's Committee.

The study participants who read the event were instructed to write on a blank page an appropriate reply to the letter and to detail as much as possible the reasons for their response.

\section{(c)) EY}

This work is licensed under a Creative Commons Attribution 3.0 License. 